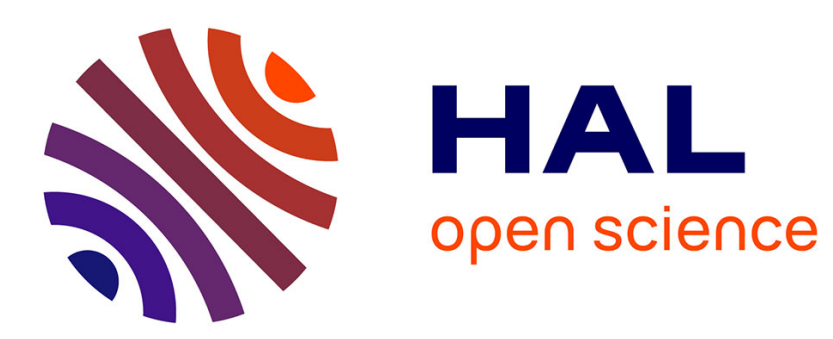

\title{
On the Laplace-Young equation applied to spherical fluid inclusions in solid matrices
}

\author{
A. Jelea
}

\section{To cite this version:}

A. Jelea. On the Laplace-Young equation applied to spherical fluid inclusions in solid matrices. Journal of Nuclear Materials, 2018, 505, pp.127-133. 10.1016/j.jnucmat.2018.03.051 . hal-02874510

\section{HAL Id: hal-02874510 https://hal.science/hal-02874510}

Submitted on 16 Jul 2020

HAL is a multi-disciplinary open access archive for the deposit and dissemination of scientific research documents, whether they are published or not. The documents may come from teaching and research institutions in France or abroad, or from public or private research centers.
L'archive ouverte pluridisciplinaire HAL, est destinée au dépôt et à la diffusion de documents scientifiques de niveau recherche, publiés ou non, émanant des établissements d'enseignement et de recherche français ou étrangers, des laboratoires publics ou privés. 


\title{
On the Laplace-Young equation applied to spherical fluid inclusions in solid matrices
}

\author{
A. Jelea* \\ Institut de Radioprotection et de Sûreté Nucléaire (IRSN), PSN/SEMIA/LPTM, BP3, 13115 \\ Saint-Paul-lez-Durance, France
}

\begin{abstract}
A continuum theory of surfaces is successfully applied to analyse a set of molecular dynamics results obtained on systems consisting of nanosize fluid bubbles trapped in a solid matrix. The equations of this theory supplied with molecular dynamics data allowed calculating the $\Gamma$ factor from the Laplace-Young equation as applied to systems of industrial interest, such as the helium bubbles that form along the ageing of some austenitic steel components of the nuclear reactors. The $\Gamma$ factor was found to have a non-linear dependency on the helium/steel interface strain. These findings are in contradiction with the implicit assumption made in some published literature considering $\Gamma$ as a constant.
\end{abstract}

Keywords: interface, helium bubble, austenitic steel, molecular dynamics

\footnotetext{
${ }^{*}$ Corresponding author.

E-mail address: andrei.jelea@irsn.fr (A. Jelea).
} 


\section{Introduction}

The last years have seen an increased interest in the theoretical study of nanosize fluid inclusions (bubbles) trapped in various solid matrices [1-7]. In most studies, the LaplaceYoung equation, as established for the fluid/fluid systems, is also used for the fluid/solid ones:

$$
P_{B}=\frac{2 \Gamma}{R_{B}}+P_{S}
$$

Here, $P_{B}$ is the pressure of the fluid in the bubble, $R_{B}$ is the bubble radius, $P_{S}$ is the pressure applied by the solid matrix on the bubble surface and the $\Gamma$ factor is often identified with the surface free energy of the fluid/solid interface (for example, see references [1-5]). However, for some authors [8-10], the $\Gamma$ factor from the Laplace-Young equation applied to systems containing solid surfaces is a surface stress rather than a surface free energy.

As shown in reference [11], the surface stress, which is an excess quantity, can be expressed through a second rank tensor, the surface excess stress tensor. In a spherical coordinate system, its components are calculated as follows:

$$
\gamma_{i j}=\frac{1}{R_{0}^{2}}\left[\int_{r_{\alpha}}^{R_{0}}\left[\sigma_{i j}(r)-\sigma_{i j}^{\alpha}(r)\right] r^{2} d r+\int_{R_{0}}^{r_{\beta}}\left[\sigma_{i j}(r)-\sigma_{i j}^{\beta}(r)\right] r^{2} d r\right] \quad i=r, \theta, \varphi
$$

In equation $2, r_{\alpha}$ and $r_{\beta}$ are the $\alpha$ and $\beta$ side limits of the $\alpha / \beta$ interface, $\sigma_{i j}(r)$ are the components of the stress tensor in the interface and $\sigma^{\alpha, \beta}{ }_{i j}(r)$ are the corresponding components of the stress tensor in the $\alpha$ and $\beta$ bulk phases extrapolated to the geometrical dividing surface. The geometrical surface position $r=R_{0}$ is chosen in an arbitrary way within the interface $\left(r_{\alpha}<\right.$ $\left.R_{0}<r_{\beta}\right)$. If the system has a spherical symmetry and, in addition, the bulk phases $\alpha$ and $\beta$ are homogeneous, the following equations hold: 


$$
\begin{gathered}
\forall r \in\left[r_{\alpha}, R_{0}\right) \quad \sigma_{\theta \theta}^{\alpha}(r)=\sigma_{\varphi \varphi}^{\alpha}(r)=\sigma_{r r}^{\alpha}(r)=\sigma_{r r}^{\alpha}\left(R_{0}\right)=\sigma_{r r}^{\alpha}\left(r_{\alpha}\right)=c t_{1} \\
\forall r \in\left\lfloor R_{0}, r_{\beta}\right\rfloor \quad \sigma_{\theta \theta}^{\beta}(r)=\sigma_{\varphi \varphi}^{\beta}(r)=\sigma_{r r}^{\beta}(r)=\sigma_{r r}^{\beta}\left(R_{0}\right)=\sigma_{r r}^{\beta}\left(r_{\beta}\right)=c t_{2} \\
\forall r \quad \sigma_{i j}^{\alpha, \beta}(r)=\sigma_{i j}(r)=0 \quad i \neq j
\end{gathered}
$$

where $c t_{1}$ and $c t_{2}$ are constant with respect to $r$.

The definition of the surface stress by equation 2 also introduces a normal component, $\gamma_{r}$, suggesting a three dimension aspect of the surface stress. As can be seen from equation 2 , every component of the surface excess stress tensor is a function of $R_{0}$, the position of the geometrical surface in the interface. For a spherical interface, the function $\gamma_{r r}\left(R_{0}\right)$ takes the following form [11]:

$$
\gamma_{r r}\left(R_{0}\right)=\frac{R_{0}\left[\sigma_{r r}^{\beta}\left(R_{0}\right)-\sigma_{r r}^{\alpha}\left(R_{0}\right)\right]}{3}+\frac{C}{R_{0}^{2}} \text { where } C<0
$$

The shape of the function $\gamma_{r r}\left(R_{0}\right)$ expressed by equation 4 shows that there is always a geometrical surface position $R_{0}=R_{s}$ such that $\gamma_{r r}\left(R_{s}\right)=0$. For this point, the geometrical surface is called the surface of tension and the surface excess stress tensor becomes bidimensional [11].

Starting from the general condition of mechanical equilibrium at a curved interface between the phases $\alpha$ and $\beta$, the author of reference [11] also derived a vector form of the Laplace-Young equation in a generalized curvilinear coordinate system. This equation is applicable to all forms of curved interfaces irrespective of the nature of the $\alpha$ and $\beta$ bulk phases (solid or fluid). If a spherical coordinate system is used and the system has a spherical symmetry, the generalized Laplace-Young vector equation reduces to the following scalar 
form:

$$
\sigma_{r r}^{\alpha}\left(R_{0}\right)-\sigma_{r r}^{\beta}\left(R_{0}\right)=-\frac{2 \Gamma}{R_{0}}
$$

In equation $5,{\sigma_{r r}}^{\alpha}\left(R_{0}\right)$ and ${\sigma_{r r}}^{\beta}\left(R_{0}\right)$ are the radial components of the stress tensor in the bulk phases $\alpha$ and $\beta$ applied to the $\alpha$ side $\left(r=r_{\alpha}\right)$ and the $\beta$ side $\left(r=r_{\beta}\right)$ faces of the interface, extrapolated to the geometrical surface $\left(r=R_{0}\right)$. In the case where the $\alpha$ and $\beta$ bulk phases are homogeneous, the equations 3 apply and the extrapolation gives: $\sigma_{r r}{ }^{\alpha}\left(R_{0}\right)=\sigma_{r r}{ }^{\alpha}\left(r_{\alpha}\right)$ and $\sigma_{r r}^{\beta}\left(R_{0}\right)=\sigma_{r r}^{\beta}\left(r_{\beta}\right) . \Gamma$ is calculated using the following relation:

$$
\Gamma=\frac{\Gamma_{\theta \theta}+\Gamma_{\varphi \varphi}}{2}
$$

where $\Gamma_{\theta \theta}$ and $\Gamma_{\varphi \varphi}$ are obtained from the tangential components of the stress tensor:

$$
\Gamma_{i i}=\frac{1}{R_{0}}\left[\int_{r_{\alpha}}^{R_{0}}\left[\sigma_{i i}(r)-\sigma_{i i}^{\alpha}(r)\right] r d r+\int_{R_{0}}^{r_{\beta}}\left[\sigma_{i i}(r)-\sigma_{i i}^{\beta}(r)\right] r d r\right] \quad i=\theta, \varphi
$$

The physical quantities $r_{\alpha, \beta}, \sigma_{i i}(r), \sigma^{\alpha, \beta}{ }_{i i}(r)$ and $R_{0}$ from equation 7 have the same meaning as in equation 2. However, the surface stresses they define, $\Gamma_{i i}$ and $\gamma_{i i}$, are different, with one exception [11], when the geometrical surface is chosen to coincide with the surface of tension $\left(R_{0}=R_{S}\right)$. Only for this case, when the surface excess stress tensor becomes bi-dimensional, the $\Gamma$ factor from the Laplace-Young equation is equal with $\gamma^{m}$, the average of the tangential components of the surface excess stress tensor:

$$
\begin{gathered}
\Gamma_{\theta \theta}\left(R_{s}\right)=\gamma_{\theta \theta}\left(R_{s}\right) \quad \Gamma_{\varphi \varphi}\left(R_{s}\right)=\gamma_{\varphi \varphi}\left(R_{s}\right) \\
\Gamma\left(R_{s}\right)=\frac{\Gamma_{\theta \theta}\left(R_{s}\right)+\Gamma_{\varphi \varphi}\left(R_{s}\right)}{2}=\frac{\gamma_{\theta \theta}\left(R_{s}\right)+\gamma_{\varphi \varphi}\left(R_{s}\right)}{2}=\gamma^{m}\left(R_{s}\right)
\end{gathered}
$$


In the present work, the concepts and relations of the continuum theory of surfaces (CTS) from reference [11] previously introduced are used to analyse a set of molecular dynamics results obtained on systems where the size of one of the phases is of the order of nanometre. For the molecular dynamics study, one chose the particular case of helium bubbles confined in a face centered cubic (fcc) FeNiCr alloy at compositions similar to AISI-316 austenitic steels: the $\alpha$ phase is the helium (He) and the $\beta$ phase is the metal (M).

This work has also an applied objective, since it provides two methods allowing to calculate the $\Gamma$ factor for fluid/solid interfaces in systems of industrial interest. In particular, predicting the evolution and the properties of the helium bubbles in AISI-316 austenitic steels is crucial for understanding the ageing phenomena affecting some steel components of the nuclear reactors.

The paper is structured as follows: Section 2 presents the models and methods employed in this study. The first part of Section 3 gives a characterization of the helium/steel interface on the basis of molecular dynamics results. In the second part of Section 3, the concepts and equations of CTS are employed to analyse the molecular dynamics data and to calculate the $\Gamma$ factor from the Laplace-Young equation. Section 4 gives a summary of the results of this study.

\section{Models and methods}

\subsection{Models for the helium/steel systems}

The calculations presented in this paper were carried out using 3D periodic boundary conditions. The pattern for the periodical model was built starting from a supercell of $31 \times 31$ x 31 fcc elementary cells. The fcc sites of this supercell were randomly filled with $\mathrm{Fe}, \mathrm{Ni}$ and 
$\mathrm{Cr}$ atoms in order to create a model reproducing the AISI-316 alloy which has a composition (in weight \%) of $12 \% \mathrm{Ni}, 17.5 \% \mathrm{Cr}$ and $70.5 \% \mathrm{Fe}$ [12] and displays a homogeneous distribution of the components $(\mathrm{Fe}, \mathrm{Ni}, \mathrm{Cr})$ in the matrix [13]. The resulting cubic supercell contains $83385 \mathrm{Fe}$ atoms, $13512 \mathrm{Ni}$ atoms and $22267 \mathrm{Cr}$ atoms.

This perfect fcc supercell was then relaxed through molecular dynamics (MD) [14] in NPT (constant Number of particles, Pressure and Temperature) ensemble at $\mathrm{T}=600 \mathrm{~K}$ and $\mathrm{P}$ $=0 \mathrm{GPa}$ (no stress in the matrix). Following this relaxation, the edge length of the supercell takes the value $a=10.962 \mathrm{~nm}$.

Cavities with different radii $\left(\mathrm{R}_{\mathrm{C}}\right)$ were carved in the relaxed $\mathrm{FeNiCr}$ fcc supercell by eliminating all the $\mathrm{Fe}, \mathrm{Ni}$ and $\mathrm{Cr}$ atoms situated in a $\mathrm{R}_{\mathrm{C}}$ radius sphere with the center in the middle of the supercell. Four values were chosen for $\mathrm{R}_{\mathrm{C}}: 1.0 \mathrm{~nm}, 1.3 \mathrm{~nm}, 1.7 \mathrm{~nm}$ and $2.0 \mathrm{~nm}$. The four cavities were then filled with different numbers of helium atoms in order to cover a range of average helium densities, from about $20 \mathrm{~nm}^{-3}$ to $110 \mathrm{~nm}^{-3}$.

\subsection{Simulation methods}

The helium/steel systems built as previously described were then relaxed at $\mathrm{T}=600 \mathrm{~K}$ using NVT (constant Number of particles, Volume and Temperature) MD methods as implemented in the LAMMPS code [15]. The NVT-MD relaxation was carried out solely on the supercell atoms contained in an $R_{b o x}$ radius sphere $\left(R_{b o x}=5 \mathrm{~nm}\right)$ with the center in the middle of the supercell. All the supercell atoms situated outside the $R_{b o x}$ radius sphere were fixed at their perfect lattice positions, thus insuring a fixed boundary for the spherical relaxed region. The thickness of the layer of fixed atoms $(\sim 1 \mathrm{~nm})$ is larger than the cutoff radius of any interatomic potential used in these simulations. Thus, the atoms from the relaxed region 
do not "see" their periodic images. Moreover, the layer of fixed atoms ensures that the stress field present in the spherical relaxed region does not interact with the stress fields of the periodic images, thus imposing a spherical symmetry.

A time step of $0.2 \mathrm{fs}$ was found appropriate for this type of simulations [2]. The systems were relaxed during $0.5 \mathrm{~ns}\left(2.5 \times 10^{6} \mathrm{MD}\right.$ steps $)$, then the results were accumulated over the next $2.5 \times 10^{6}$ MD steps at every 100 steps. Thus, for every quantity of interest, one obtained a set of 25000 instantaneous values. The properties of interest (stress tensor components, energies and bubble radii) were then calculated as averages of these instantaneous values.

The stress tensor components in a certain region of the system were computed from the atomic stress tensor components of the atoms contained in this region (provided by LAMMPS in pressure*volume units [15]) and the volume of the region.

\subsection{Interatomic potentials}

In all simulations, semi empirical interatomic potentials were employed to describe the three types of interactions that are present in the systems: Fe-Ni-Cr, $\mathrm{M}-\mathrm{He}(\mathrm{M}=\mathrm{Fe}, \mathrm{Ni}, \mathrm{Cr})$ and He-He.

For the Fe-Ni-Cr interactions, one used a ternary embedded atom method (EAM) type potential [16] which is able to give good estimations, with respect to Density Functional Theory (DFT) and experimental results, for some important properties of the AISI-316L austenitic steels: the stability of the fcc phase, the elastic constants and the stacking fault energies.

The $\mathrm{Fe}-\mathrm{He}$ and $\mathrm{Cr}-\mathrm{He}$ interactions were described using the potentials proposed in 
reference [17]. These potentials, based on the pair potential formalism, were fitted in order to correctly reproduce, with respect to DFT results, the migration energies of helium in Fe and $\mathrm{Cr}$ and the formation energy of the substitutional and interstitial helium in the tetrahedral and octahedral sites of the $\mathrm{Fe}$ and $\mathrm{Cr}$ matrices.

The potential used for the $\mathrm{Ni}-\mathrm{He}$ interaction was fitted to a set of $\mathrm{Ni}-\mathrm{He}$ interaction energies obtained by Melius [18] using the Hartree-Fock approximation. The form of this potential is given below:

$$
V(r)=\left\{\begin{array}{cl}
\left(A+\frac{B}{r}+\frac{C}{r^{2}}\right) e^{-D r} & r<r_{i} \\
P_{3} r^{3}+P_{2} r^{2}+P_{1} r+P_{0} & r_{i} \leq r \leq r_{c} \\
0 & r>r_{c}
\end{array}\right.
$$

The potential parameters, together with the cutoff radii, are shown in Table 1.

For the He-He interactions, one employed the potential proposed by Ross and Young [19] that reasonably reproduces (at least at $\sim 300 \mathrm{~K}$ ), with respect to DFT and experimental data [20], the pressure versus density curve for helium.

\section{Results and discussion}

\subsection{The helium/steel interface}

Figure 1 schematically represents a cross section of the systems analysed here. The relaxed region is formed of three spherical sub regions: the bulk helium region $\left(0<r<r_{H e}\right)$, the helium/steel (metal) interface region $\left(r_{H e}<r<r_{M}\right)$ and the bulk metal region $\left(r_{M}<r<\right.$ $\left.R_{b o x}\right)$. The limits of the helium/metal interface region, $r_{H e}$ and $r_{M}$, were determined in each of 
the studied systems using the fact that at the interface the intensive properties diverge from those in the bulk. Figure 2 represents a typical radial profile of the potential energy per atom as obtained from the MD data. The profile displays a discontinuity at the interface around which the potential energy differs from the constant bulk values. This variation of the potential energy is present on both helium and metal sides of the interface, but on the metal side the variation is steeper, allowing to easily distinguish between the interface and the bulk metal. Thus, the point $\left(r=r_{M}\right)$ from which the potential energy per atom starts to diverge from the bulk value (-4.1 eV/atom) is the limit of the interface on the metal side. On the helium side, the potential energy variation is less steep. Moreover, for some systems with low helium densities, the statistics in many spherical regions on the helium side is very poor, generating strong fluctuations in the potential energy profile that further complicate the identification of the interface/bulk limit. However, another criterion allows identifying this limit: the radial profiles of the three diagonal components of the stress tensor. As can be seen in Figure 3, the three diagonal components are identical in the bulk helium region, as expected for a fluid, but starting from a certain point this symmetry tends to be broken: the two tangential components, $\sigma_{\theta \theta}(r)$ and $\sigma_{\varphi \varphi}(r)$, are still equal among themselves but different from the radial component $\sigma_{r r}(r)$. The point $\left(r=r_{H e}\right)$ from which the symmetry breaking occurs is the limit of the interface on the helium side.

The interface width values for all the studied systems, as calculated from the interface limits, are represented on Figure 4. These values range from $0.8 \mathrm{~nm}$ to $1.1 \mathrm{~nm}$, in good agreement with other estimations from the published literature [21].

$R_{B}$ from Figure 1 represents the bubble radius. It was calculated as the arithmetic mean between a "cavity minimum radius" and a "cluster maximum radius". The "cavity minimum radius" was defined as the distance between the bubble mass center and the closest matrix 
atom ( $\mathrm{Fe}, \mathrm{Ni}$ or $\mathrm{Cr}$ ) and the "cluster maximum radius" as the distance between the bubble mass center and the furthest (helium) atom in the helium cluster. Following this definition [22], the bubble surface $\left(r=R_{B}\right)$ would be situated at the crossing point of the matter densities of the two phases.

\subsection{Calculation of $\boldsymbol{\Gamma}$ from molecular dynamics data}

Figure 5 displays an example of a typical radial profile, obtained through MD simulations, for the components of the stress tensor in the relaxed spherical region from the supercell. Every point represents the time average of the stress component calculated in a $0.01 \mathrm{~nm}$ thick shell situated at the distance $r$ from the center of the spherical region.

The stress tensor is diagonal everywhere $\left(\sigma_{i j}=0, i \neq j\right)$ as a consequence of the spherical symmetry. In the bulk helium region, the three diagonal components, which are identical, oscillate around a constant value, $-P_{H e}$, where $P_{H e}$ is the helium hydrostatic pressure. In the bulk metal region, all three diagonal components of the stress tensor diminish in intensity with increasing $r$ while, as shown on Figure 6, the pressure profile displays small fluctuations around a constant value.

From the viewpoint of solid mechanics, the systems analysed here correspond to the case of an $R_{b o x}$ radius solid sphere (bulk metal) containing an $r_{M}$ radius spherical inclusion (bulk helium plus the interface). The spherical inclusion exerts a pressure $P_{\text {int }}$ on its borders $(r=$ $\left.r_{M}\right)$, which translates into a pressure $P_{e x t}$ at the (fixed) outer border of the solid sphere $(r=$ $\left.R_{b o x}\right)$. For this situation, the stress tensor is diagonal and its diagonal components in the solid sphere have the following form [23]: 


$$
\begin{gathered}
\sigma_{r r}(r)=-\frac{R_{b o x}^{3} r_{M}^{3}\left(P_{\text {int }}-P_{e x t}\right)}{\left(R_{b o x}^{3}-r_{M}^{3}\right) r^{3}}+\frac{r_{M}^{3} P_{\text {int }}-R_{b o x}^{3} P_{e x t}}{R_{b o x}^{3}-r_{M}^{3}} \\
\sigma_{\theta \theta}(r)=\sigma_{\varphi \varphi}(r)=\frac{R_{b o x}^{3} r_{M}^{3}\left(P_{\text {int }}-P_{e x t}\right)}{2\left(R_{b o x}^{3}-r_{M}^{3}\right) r^{3}}+\frac{r_{M}^{3} P_{\text {int }}-R_{b o x}^{3} P_{e x t}}{R_{b o x}^{3}-r_{M}^{3}}
\end{gathered}
$$

The pressure in the solid sphere $\left(P_{M}\right)$ is obtained from the trace of the stress tensor:

$$
P_{M}=-\frac{1}{3} \operatorname{Tr}[\underline{\underline{\sigma}}]=\frac{r_{M}^{3} P_{\mathrm{int}}-R_{b o x}^{3} P_{e x t}}{r_{M}^{3}-R_{b o x}^{3}}
$$

According to equations 10 and 11, the stress tensor diagonal components in the bulk metal exhibit a $1 / r^{3}$ decrease, while the pressure is constant everywhere, in agreement with the MD results. Moreover, if the values of $P_{i n t}, P_{\text {ext }}$ and $P_{M}$ are calculated through MD and introduced in equations 10 and 11, the resulting analytical functions (solid lines from Figures 5 and 6) fit very well the MD points.

Considering that the helium and metal bulk phases are homogeneous and given that $\sigma_{r r}{ }^{M}\left(r_{M}\right)=-P_{\text {int }}$ (it follows from the first equation 10) and $\sigma_{r r}{ }^{H e}\left(r_{H e}\right)=-P_{H e}($ the bulk helium is a fluid), the equation 5, as applied to helium/metal systems, takes the following familiar Laplace-Young form:

$$
P_{H e}=\frac{2 \Gamma}{R_{B}}+P_{\text {int }}
$$

In the above equation, the geometrical dividing surface was chosen to coincide with the bubble surface $\left(R_{0}=R_{B}\right)$.

It should be noted that, despite their formal identity, the equations 1 and 12 contain terms with a different meaning. For example, $P_{B}$ from equation 1 usually represents the pressure in the whole volume of the fluid bubble, while $P_{H e}$ from equation 12 refers only to the bulk fluid 
region. Both pressures are practically identical for the large bubbles, but for the very small ones, where the interface width $(\sim 1 \mathrm{~nm})$ is of the same order of magnitude as the bubble size, the difference between $P_{H e}$ and $P_{B}$ could be important. Moreover, from equation 12, which is only a particular form of equation 5 , the nature of $\Gamma$ as a surface stress like quantity now becomes obvious.

Equation 12 gives an easy access to the value of $\Gamma$, since $P_{H e}, R_{B}$ and $P_{\text {int }}$ can all be computed with MD techniques. The MD calculated radial profiles for the components of the stress tensor also allow determining the value of $\Gamma$ by using its definition (equation 6 ) where the tangential components are calculated through a discretised form of equation 7 , as follows:

$$
\begin{gathered}
\Gamma_{i i}=\frac{1}{R_{0}}\left(I_{i i}^{1}+I_{i i}^{2}\right) \quad i=\theta, \varphi \\
I_{i i}^{1} \approx \sum_{r_{H e}<r_{i} \leq R_{0}} \sigma_{i i}\left(r_{i}\right) r_{i} \Delta r+\frac{P_{H e}\left(R_{0}^{2}-r_{H e}^{2}\right)}{2} \quad \Delta r=0.01 n m \\
I_{i i}^{2} \approx \sum_{R_{0}<r_{i} \leq r_{M}} \sigma_{i i}\left(r_{i}\right) r_{i} \Delta r+\frac{P_{\mathrm{int}}\left(r_{M}^{2}-R_{0}^{2}\right)}{2} \quad \Delta r=0.01 \mathrm{~nm}
\end{gathered}
$$

Figure 7 shows that, for all the studied systems, the values of $\Gamma$ calculated with equations 13 when $R_{O}=R_{B}\left(\Gamma_{D E F}\right)$ and equation $12\left(\Gamma_{L A}\right)$ are practically identical. The agreement between $\Gamma_{D E F}$ and $\Gamma_{L A}$ proves the validity of the CTS from reference [11] when applied to our systems in the nanometer size range.

As discussed in section 2.1, the helium/metal systems were built by filling, with increasing amounts of helium, void cavities with four different radii $R_{C}$ carved in the metal matrix. The MD relaxation of a void cavity leads to a partial shrink of its volume. When helium is gradually added, the pressure it creates counterbalances this shrinking tendency by pushing 
the cavity walls outwards and increasing again the cavity volume. The bubble radius $\left(R_{B}\right)$ is thus an increasing function of the number of helium atoms in the bubble $\left(N_{H e}\right)$. The $R_{B}\left(N_{H e}\right)$ variations corresponding to the four families of helium/metal systems can be well-fitted with linear functions (see Figure 8). As a result of the $R_{B}$ variation, the interface is deformed and the associated strain $\left(\varepsilon_{s}\right)$ can be defined as follows:

$$
\varepsilon_{s}=\frac{R_{B}-R_{B}^{0}}{R_{B}^{0}} \text { where } R_{B}^{0}=\lim _{N_{H e} \rightarrow 0} R_{B}\left(N_{H e}\right)
$$

As shown on Figure 9, the interface strain has a strong impact on the $\Gamma$ value: $\Gamma$ is a quadratic (or a higher order) polynomial function of $\varepsilon_{s}$. One should mention that the surface strain dependency of $\Gamma$ has already been considered by some authors $[9,10]$, albeit in a linear form, which is appropriate for small surface deformations.

The curves $\Gamma\left(\varepsilon_{s}\right)$, which are different from a bubble family to another, suggest different surface elastic properties for the four cases. This is explained by the fact that the four cavities carved in the metal matrix are faceted and the nature and proportion of facets composing them are different from a cavity to another. As the elastic properties of the bubble surface depend on the nature and proportion of facets, they are also different from a bubble family to another. This faceting effect is strong in small bubbles and one expects it to vanish for the large ones. As a consequence, the $\Gamma\left(\varepsilon_{s}\right)$ curves would become practically identical for large helium filled cavities.

All the previous $\Gamma$ values were determined for the case where the geometrical surface coincides with the bubble surface $\left(R_{0}=R_{B}\right)$. However, the geometrical surface position can be chosen anywhere between the interface limits as stated in section 1. Changing the position of the geometrical surface has an impact on the values of the excess quantities. Figure 10 shows, for one of the studied systems, the variations of $\Gamma, \gamma^{m}$ (the average of the tangential 
components of the surface excess stress tensor, $\gamma_{\theta \theta}$ and $\gamma_{\varphi \varphi}$ ) and $\gamma_{r r}$ (the normal component of the surface excess stress tensor) with $R_{0}$. The components of the surface excess stress tensor $\left(\gamma_{i i}\right)$ were calculated from MD data using a discretised form of equation 2:

$$
\begin{gathered}
\gamma_{i i}=\frac{1}{R_{0}^{2}}\left(T_{i i}^{1}+T_{i i}^{2}\right) \quad i=r, \theta, \varphi \\
T_{i i}^{1} \approx \sum_{r_{H e}<r_{i} \leq R_{0}} \sigma_{i i}\left(r_{i}\right) r_{i}^{2} \Delta r+\frac{P_{H e}\left(R_{0}^{3}-r_{H e}^{3}\right)}{3} \quad \Delta r=0.01 n m \\
T_{i i}^{2} \approx \sum_{R_{0}<r_{i} \leq r_{M}} \sigma_{i i}\left(r_{i}\right) r_{i}^{2} \Delta r+\frac{P_{\text {int }}\left(r_{M}^{3}-R_{0}^{3}\right)}{3} \quad \Delta r=0.01 n m
\end{gathered}
$$

The $\gamma_{r r}$ component cancels at the point where the geometrical surface coincides with the surface of tension $\left(R_{0}=R_{s}\right)$. Figure 11 shows that, for all the studied systems, the values of $\Gamma\left(R_{0}=R_{S}\right)$ and $\gamma^{m}\left(R_{0}=R_{S}\right)$ practically coincide, that is, the $\Gamma\left(R_{0}\right)$ and $\gamma^{m}\left(R_{0}\right)$ curves cross each other at the surface of tension, as predicted by the CTS.

\section{Conclusions}

In the present study, some concepts and equations of a continuum theory of surfaces were successfully employed to analyse the MD simulation results obtained on systems consisting of nanosize helium bubbles embedded in a solid matrix with the same structure and composition as the AISI-316 austenitic stainless steel. These systems are of particular interest to the nuclear industry.

By showing a very good agreement between the MD results and the continuum theory predictions, this study also allowed to determine, through MD simulation techniques, the $\Gamma$ factor from the Laplace-Young equation as applied to nanosize helium/steel systems. More 
precisely, $\Gamma$ was calculated from MD supplied results by two simple methods: using the Laplace-Young equation itself or through the definition of $\Gamma$ as an excess quantity.

It was shown that $\Gamma$, which is a surface stress like quantity, has a non-linear dependency on the interface strain, that is, the interface behaves like an elastic membrane. Despite the fact that some authors took into account the interface strain dependency of the $\Gamma$ factor in the Laplace-Young equation applied to solid interfaces, in many papers $\Gamma$ is still implicitly considered to be a constant, the value of which coincides with that of the surface free energy.

\section{Acknowledgements}

All the calculations were performed using the IRSN computing resources. I would like to thank Dr. P.-G. Vincent from IRSN/SEMIA/LPTM for his useful remarks on some mechanical aspects of this study.

\section{Data availability}

The raw/processed data required to reproduce these findings cannot be shared at this time as the data also forms part of an ongoing study. 


\section{References}

[1] A. Caro, D. Schwen, J. Hetherly, E. Martinez, The capillarity equation at the nanoscale: Gas bubbles in metals, Acta Mater. 89 (2015) 14-21.

[2] R.E. Stoller, Y.N. Osetsky, An atomistic assessment of helium behavior in iron, J. Nucl. Mater. 455 (2014) 258-262.

[3] J. Cui, M. Li, J. Wang, Q. Hou, Molecular dynamics study of helium bubble pressure in tungsten, Nucl. Instr. Meth. Phys. Res. B 352 (2015) 104-106.

[4] T. Jourdan, J.-P. Crocombette, A variable-gap model for calculating free energies of helium bubbles in metals, J. Nucl. Mater. 418 (2011) 98-105.

[5] M.S. Veshchunov, On the theory of fission gas bubble evolution in irradiated $\mathrm{UO}_{2}$ fuel, $\mathrm{J}$. Nucl. Mater. 277 (2000) 67-81.

[6] J. Wang, Q. Hou, T. Sun, X. Long, X. Wu, S. Luo, Defect behavior induced by helium cluster growth in titanium crystals, J. of Appl. Phys. 102 (2007) 093510:1-4.

[7] T.X. Feng, L.C. Sheng, Z.Z. He, G. Tao, Molecular dynamics simulation of collective behaviour of Xe in $\mathrm{UO}_{2}$, Chin. Phys. B 19 (2010) 057102:1-6.

[8] R.C. Cammarata, Surface and Interface Stress Effects in Thin Films, Prog. In Surf. Sci. 46 (1994) 1-38.

[9] W.G. Wolfer, Elastic properties of surfaces on nanoparticles, Acta Mater. 59 (2011) 77367743.

[10] F.D. Fischer, J. Svoboda, Stresses in hollow nanoparticles, Int. J. Solids Struct. 47 (2010) 2799-2805. 
[11] A.I. Rusanov, Surface thermodynamics revisited, Surf. Sci. Rep. 58 (2005) 111-239.

[12] R.L. Plaut, C. Herrera, D.M. Escriba, P.R. Rios, A.F. Padilha, A Short Review on Wrought Austenitic Stainless Steels at High Temperatures: Procession, Microstructure, Properties and Performance, Materials Research 10 (2007) 453-460.

[13] J.B. Piochaud, Ph.D. Thesis, Modelling of Radiation Induced Segregation in Austenitic Fe Alloys at the Atomistic Level, Université des Sciences et Technologies de Lille, 2013.

[14] A.R. Leach, Molecular Modelling Principles and Applications, Pearson Education Limited, Harlow, 2001.

[15] S. Plimpton, Fast Parallel Algorithms for Short-Range Molecular Dynamics, J. Comput. Phys. 117 (1995) 1-19.

[16] G. Bonny, D. Terentyev, R.C. Pasianot, S. Poncé, A. Bakaev, Interatomic potential to study plasticity in stainless steels: the FeNiCr model alloy, Modelling Simul. Mater. Sci. Eng. 19 (2011) 085008: 1-14.

[17] D. Terentyev, N. Juslin, K. Nordlund, N. Sandberg, Fast three dimensional migration of He clusters in bcc Fe and Fe-Cr alloys, J. of Appl. Phys. 105 (2009) 103509: 1-12.

[18] C.F. Melius, C.L. Bisson, W.D. Wilson, Quantum-chemical and lattice-defect hybrid approach to the calculation of defects in metals, Phys. Rev. B 18 (1978) 1647-1657.

[19] M. Ross, D.A. Young, Helium at High Density, Phys. Lett. A 118 (1986) 463-466.

[20] L. Koci, R. Ahuja, A.B. Belonoshko, B. Johansson, Study of the high-pressure helium phase diagram using molecular dynamics, J. Phys.: Condens. Matter. 19 (2007) 016206: 1-9. 
[21] A. Caro, D. Schwen, E. Martinez, Structure of nanoscale gas bubbles in metals, Appl. Phys. Lett. 103 (2013) 213115: 1-4.

[22] A. Jelea, R.J.-M. Pellenq, F. Ribeiro, An atomistic modeling of the xenon bubble behavior in the $\mathrm{UO}_{2}$ matrix, J. Nucl. Mater. 444 (2014) 153-160.

[23] A.F. Bower, Applied Mechanics of Solids, CRC Press, Boca Raton (FL), 2010. 


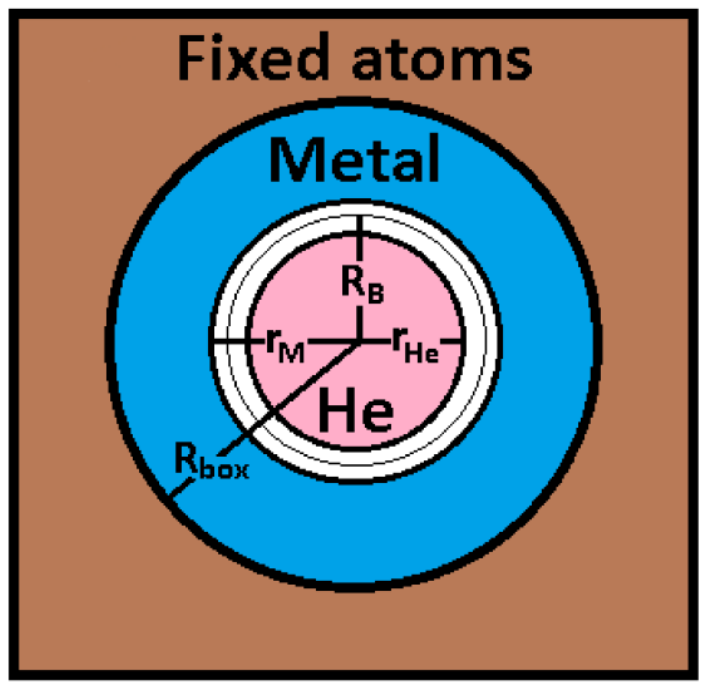

Fig. 1 


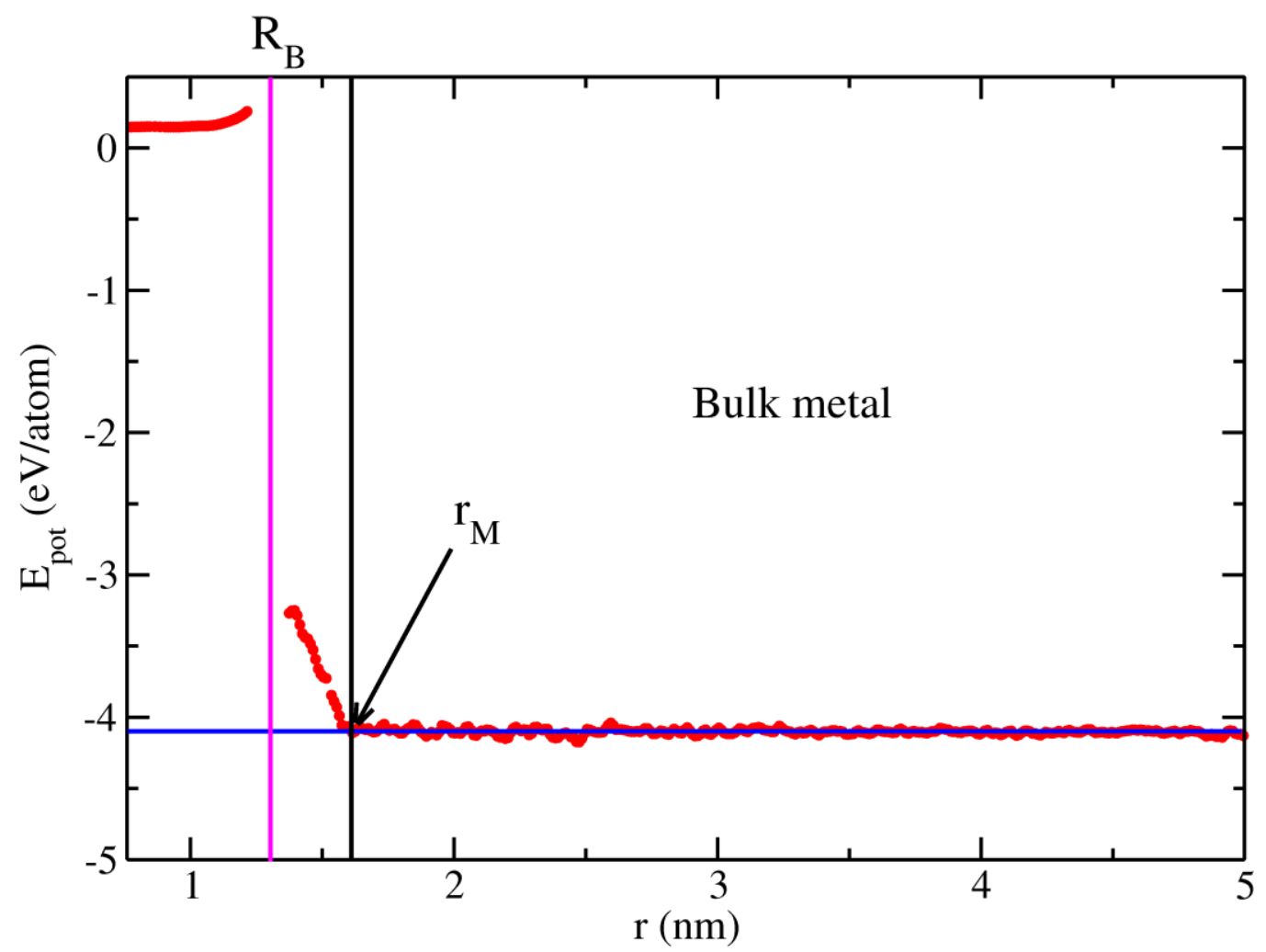

Fig. 2 


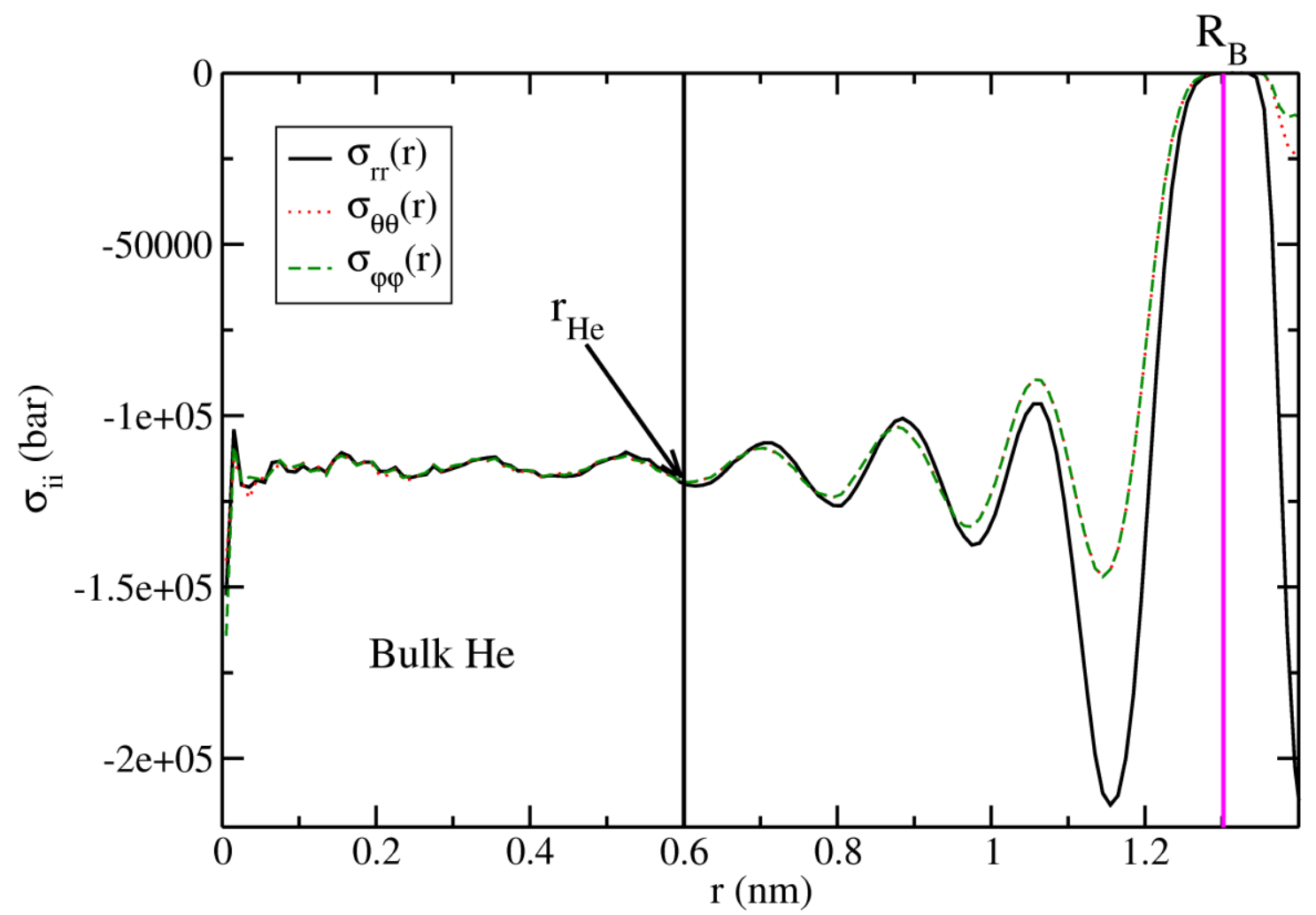

Fig. 3 


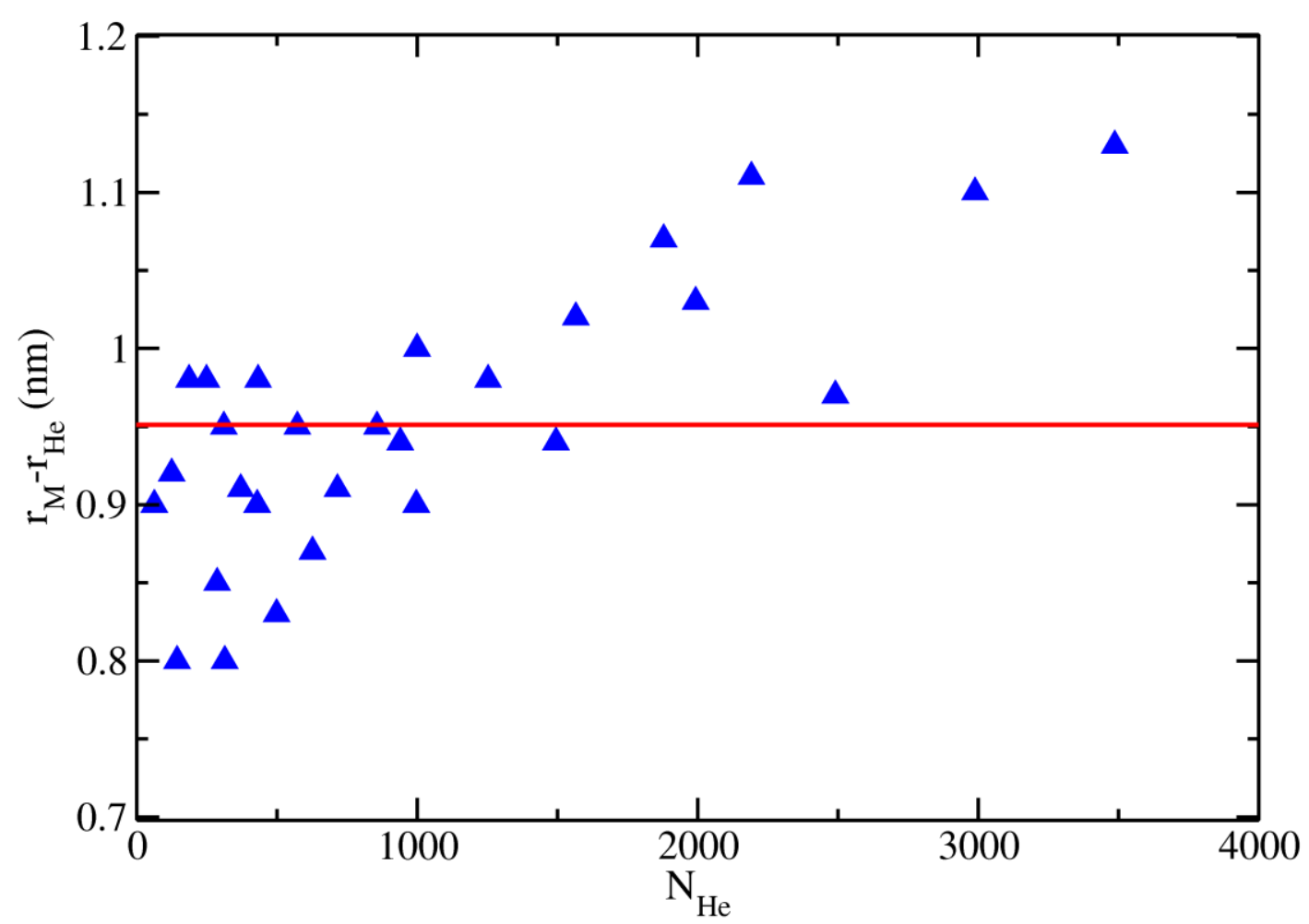

Fig. 4 


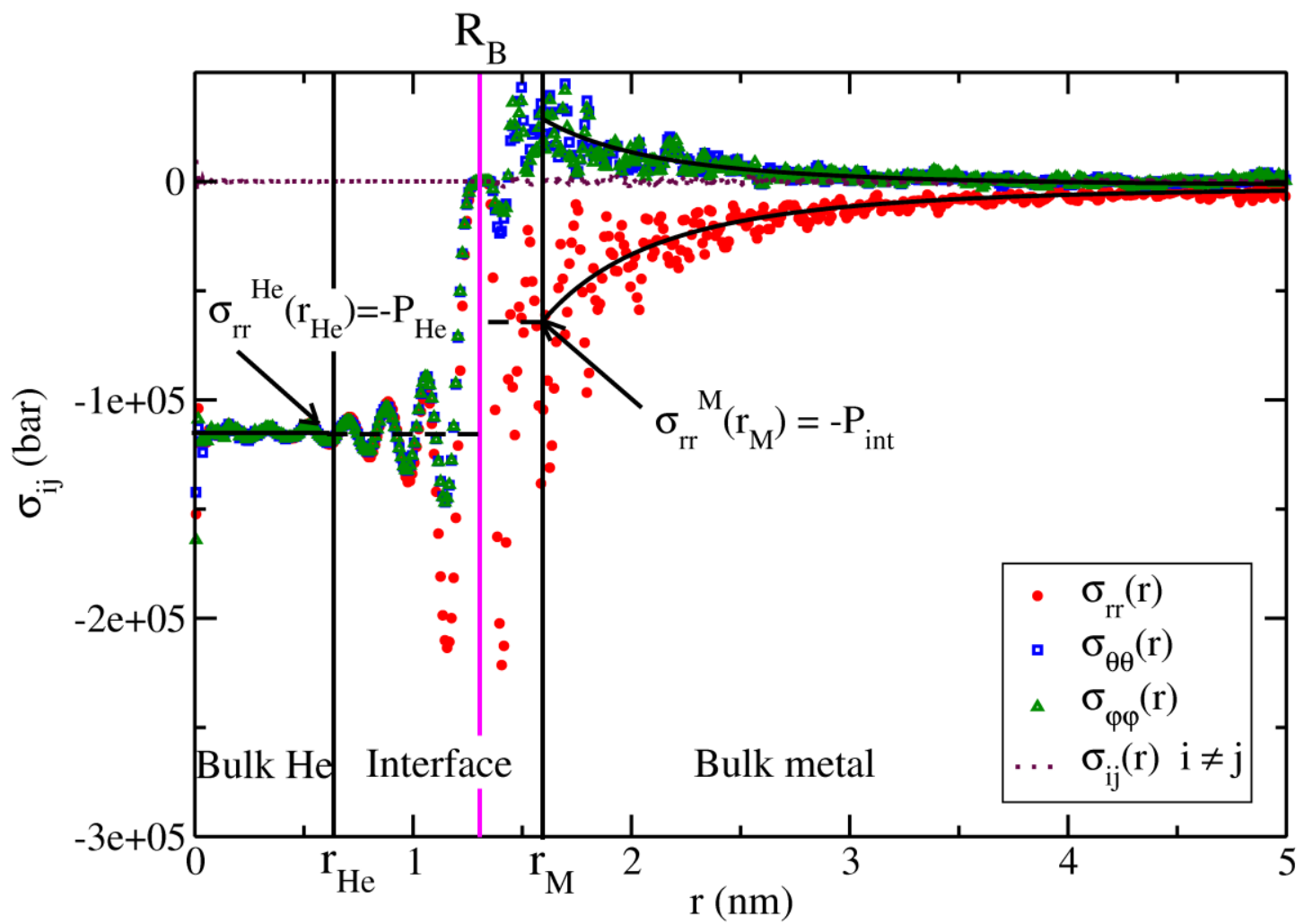

Fig. 5 


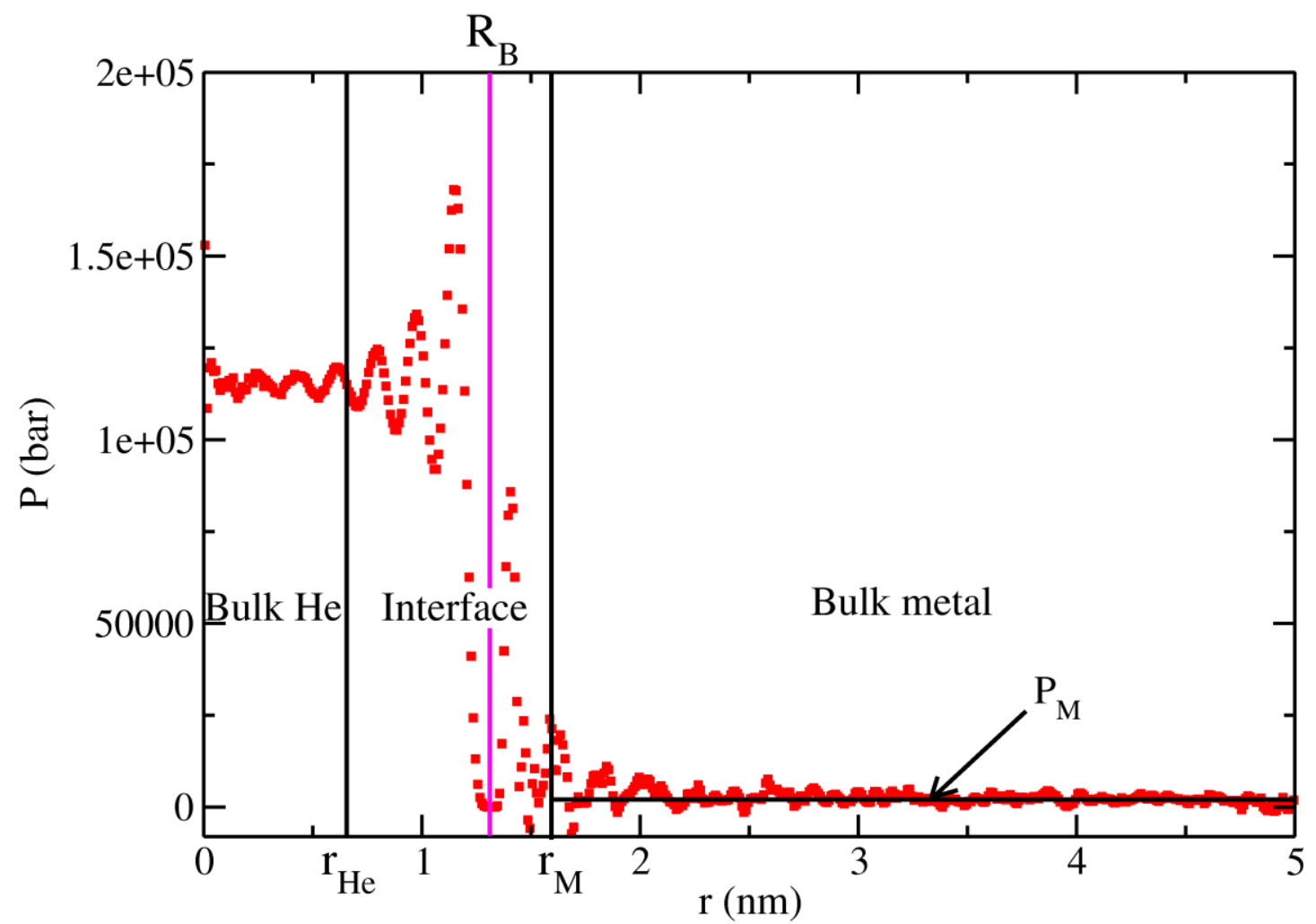

Fig. 6 


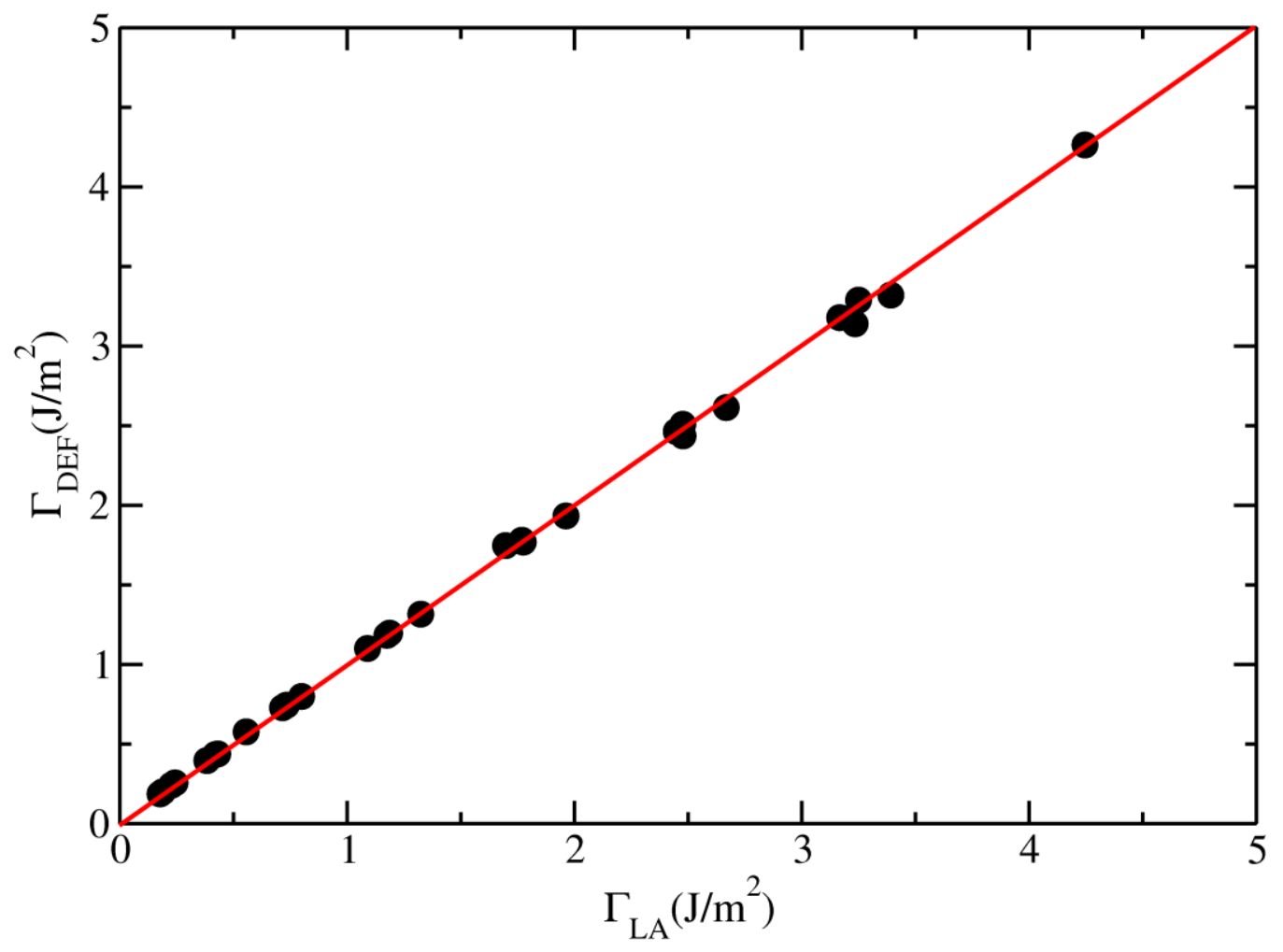

Fig. 7 


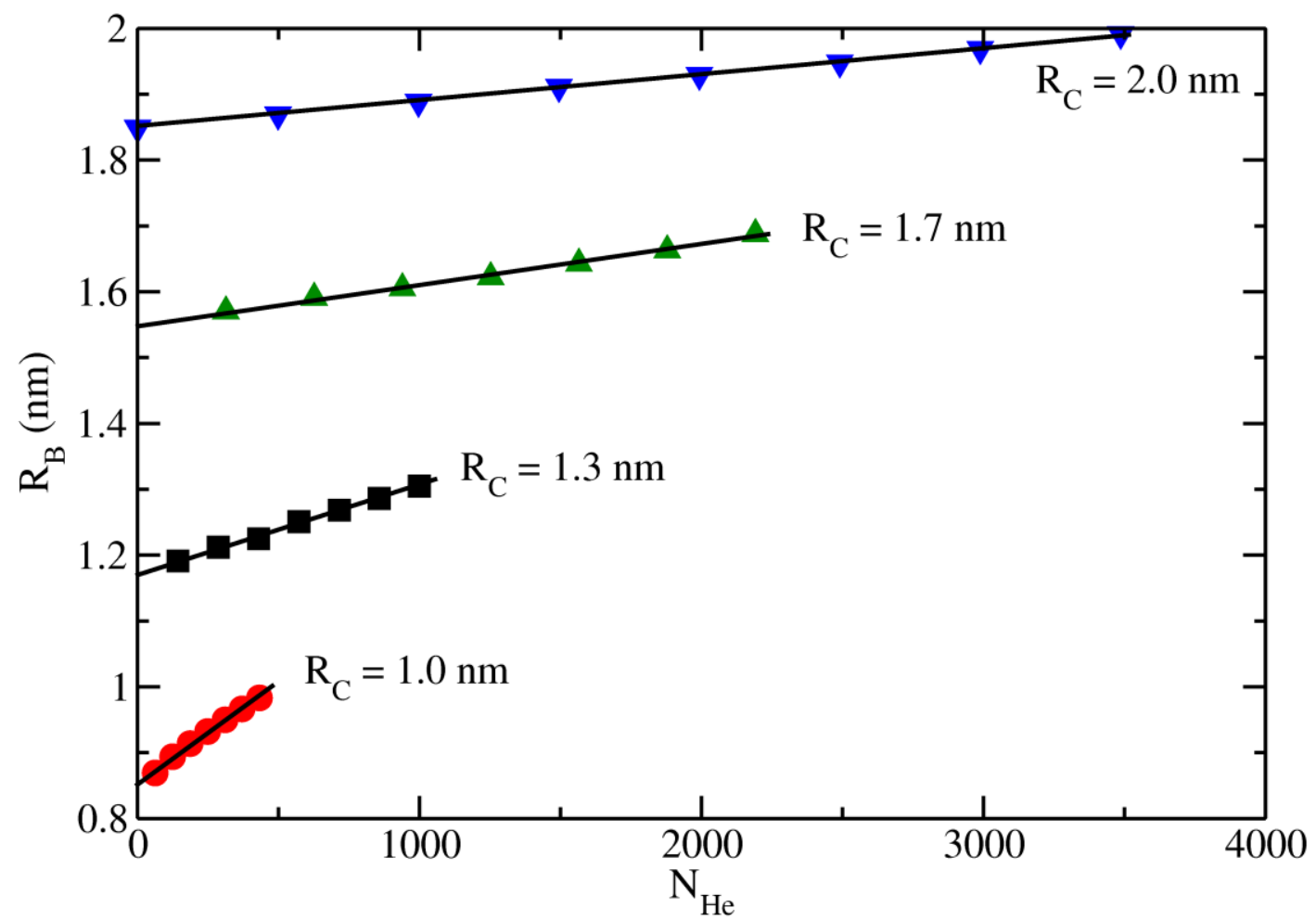

Fig. 8 


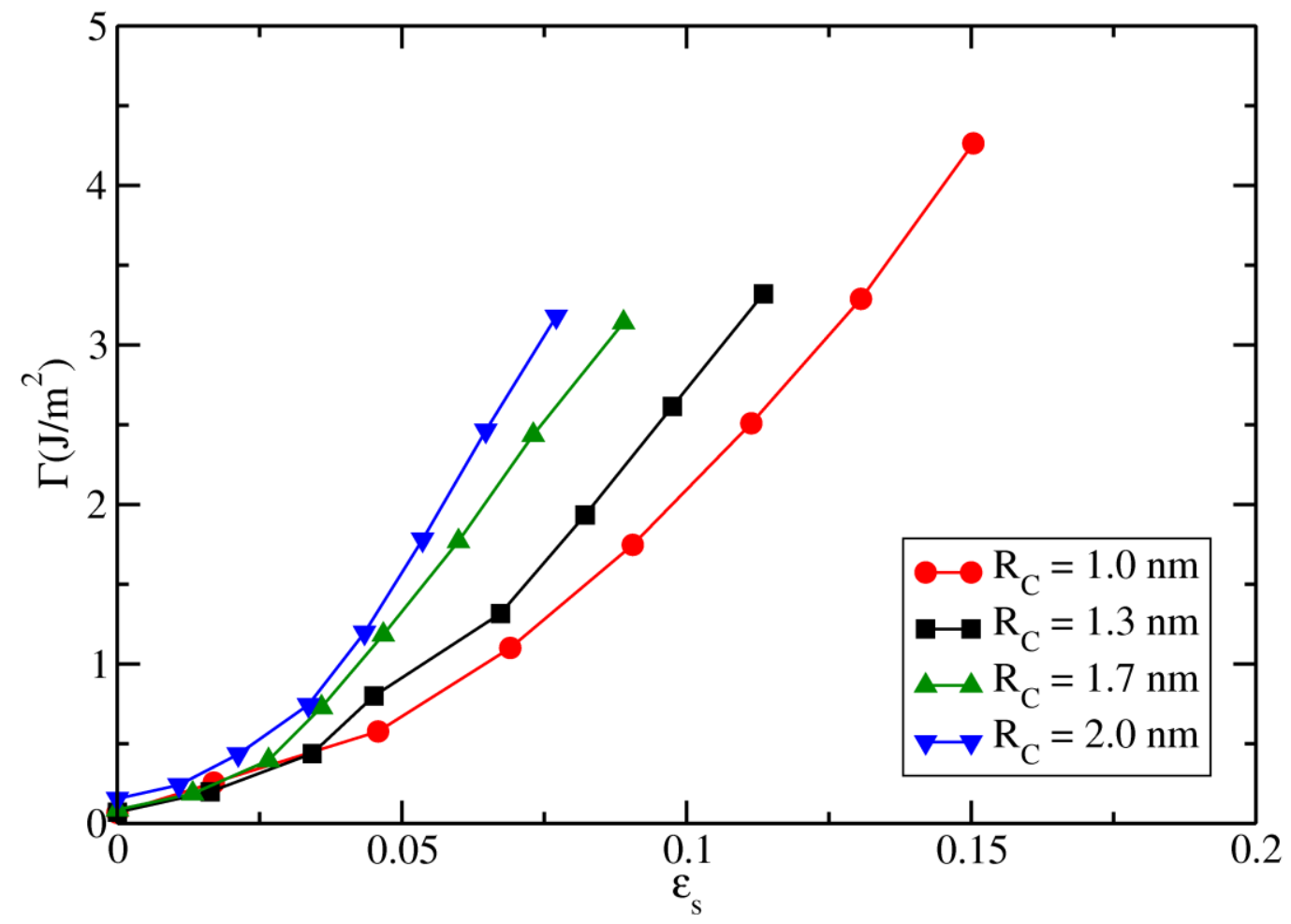

Fig. 9 


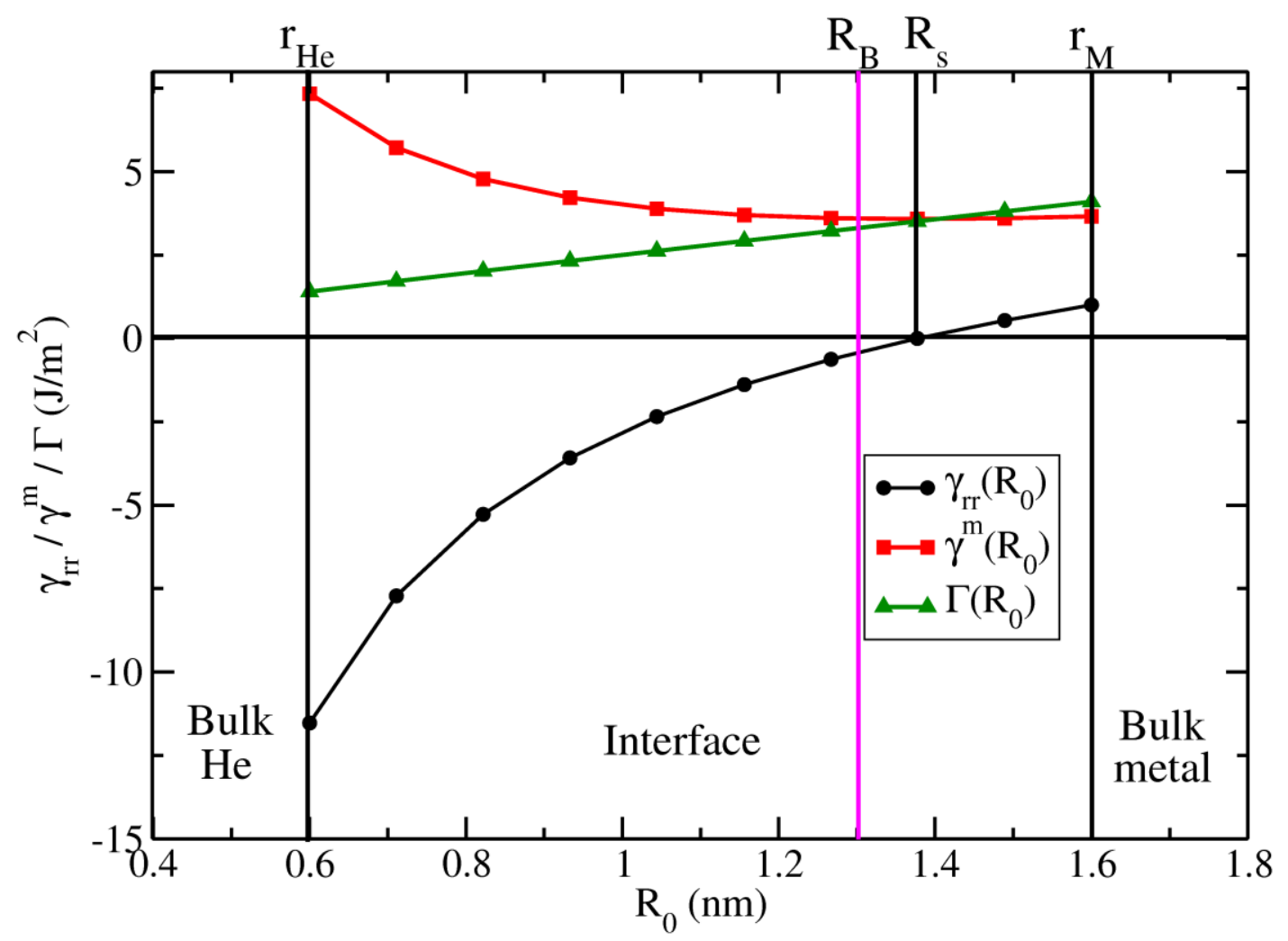

Fig. 10 


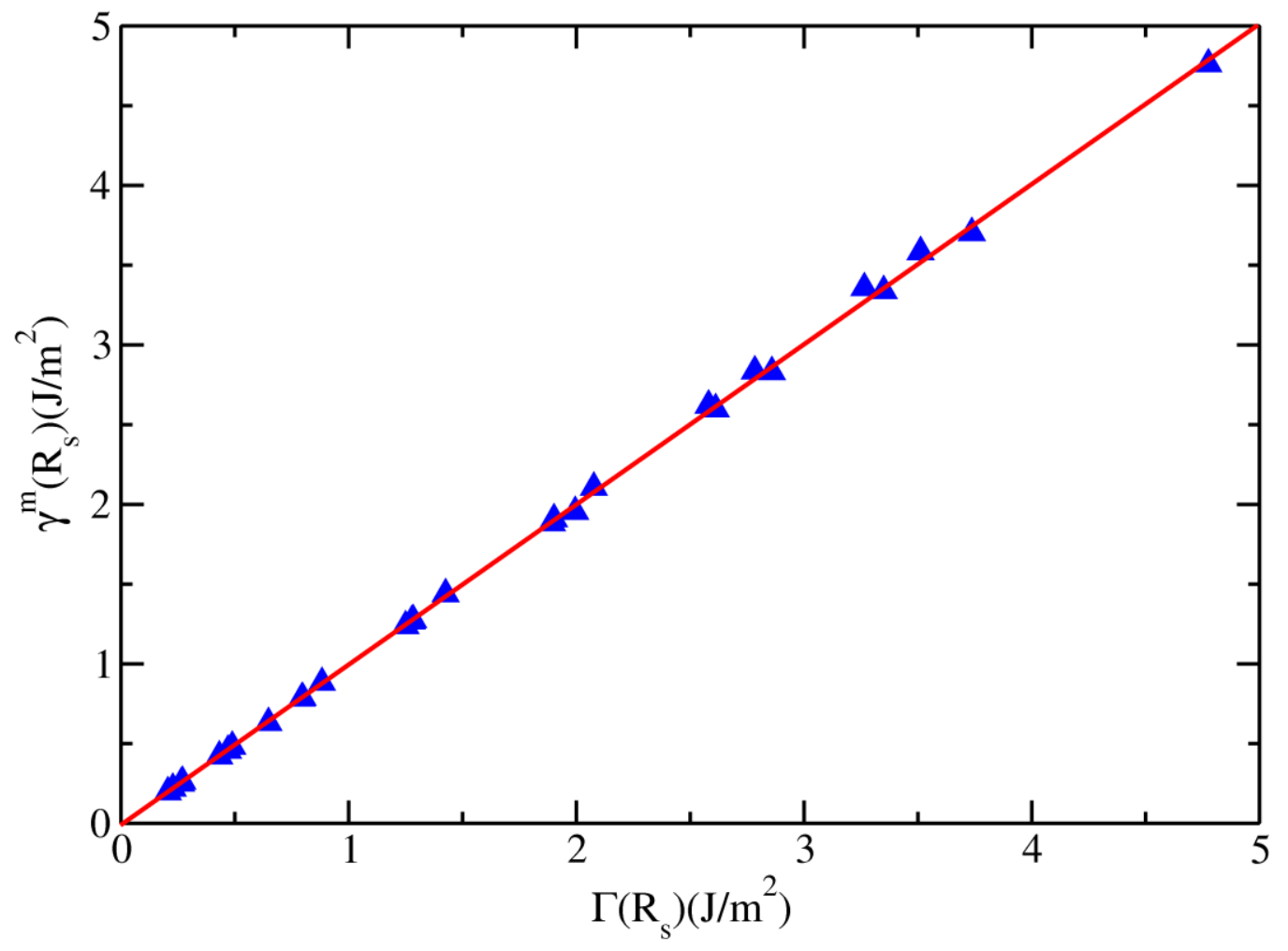

Fig. 11 


\section{Figure captions}

Fig. 1. Schematic representation of the cross-section of a supercell, such as those used in the present study.

Fig. 2. Radial profile of the potential energy per atom (full circles) in the helium/steel systems. Example corresponding to a system built from a $1.3 \mathrm{~nm}$ radius cavity filled with 999 helium atoms.

Fig. 3. Radial profiles of the diagonal components of the stress tensor in the bulk helium and at the helium/steel interface. Example corresponding to a system built from a $1.3 \mathrm{~nm}$ radius cavity filled with 999 helium atoms.

Fig.4. Helium/steel interface widths $\left(r_{M}-r_{H e}\right)$ for all the systems analysed in this work.

Fig.5. Radial profiles of the stress tensor components in the relaxed region $\left(r<R_{b o x}\right)$ of the supercell. Example corresponding to a system built from a $1.3 \mathrm{~nm}$ radius cavity filled with 999 helium atoms.

Fig.6. Radial profile of the pressure in the relaxed region $\left(r<R_{b o x}\right)$ of the supercell. Example corresponding to a system built from a $1.3 \mathrm{~nm}$ radius cavity filled with 999 helium atoms.

Fig.7. Comparison between the $\Gamma$ values calculated using the Laplace-Young equation $\left(\Gamma_{L A}\right)$ and those calculated using the definition of $\Gamma$ as an excess quantity $\left(\Gamma_{D E F}\right)$.

Fig.8. Bubble radius $\left(R_{B}\right)$ versus number of helium atoms in the bubble $\left(N_{H e}\right)$ corresponding to the four families of helium/steel systems analysed in this work.

Fig.9. $\Gamma$ versus $\varepsilon_{s}$, the interface strain, for the four families of systems analysed in the present work. 
Fig.10. $\Gamma, \gamma^{m}$ and $\gamma_{r r}$ as functions of $R_{0}$, the position of the geometrical dividing surface in the interface. The example from the figure corresponds to a system built from a $1.3 \mathrm{~nm}$ radius cavity filled with 999 helium atoms.

Fig.11. Comparison between the values of $\Gamma$ and $\gamma^{m}$ corresponding to the surface of tension $\left(R_{0}=R_{s}\right)$ 


\section{Table 1}

Parameters and cutoff radii for the Ni-He potential used in the present work. The unit for energy is the electronvolt (eV) and the unit for distance is the angstrom $(\AA)$.

\begin{tabular}{ccccc}
\hline$A(\mathrm{eV})$ & $B(\mathrm{eV} \cdot \AA)$ & $C\left(\mathrm{eV} \cdot \AA^{2}\right)$ & $D\left(\AA^{-1}\right)$ & $r_{i}(\AA)$ \\
\hline 349.732 & -917.546 & 727.518 & 2.54144 & 4.0 \\
\hline$P_{0}(\mathrm{eV})$ & $P_{l}\left(\mathrm{eV} \cdot \AA^{-1}\right)$ & $P_{2}\left(\mathrm{eV} \cdot \AA^{-2}\right)$ & $P_{3}\left(\mathrm{eV} \cdot \AA^{-3}\right)$ & $r_{c}(\AA)$ \\
\hline $3.71640 \mathrm{E}-1$ & $-2.01704 \mathrm{E}-1$ & $3.60846 \mathrm{E}-2$ & $-2.12190 \mathrm{E}-3$ & 5.0 \\
\hline
\end{tabular}

